\title{
Serum level of free thyroxine is an independent risk factor for non-alcoholic fatty liver disease in euthyroid people
}

\author{
Cuiping Shao ${ }^{1}$, Qiushi Cheng ${ }^{2}$, Shuhui Zhang ${ }^{3}$, Xinxin Xiang ${ }^{4}$, Youqing Xu' ${ }^{1}$ \\ ${ }^{1}$ Department of Gastroenterology, Beijing Tiantan Hospital, Capital Medical University, Beijing, China; ${ }^{2}$ Department of Health Care, Qingdao \\ Municipal Hospital (East), Qingdao, China; ${ }^{3}$ Department of Gastroenterology, Zaozhuang City Center District People's Hospital, Zaozhuang, \\ China; ${ }^{4}$ Center of Translational Medicine, Zibo Central Hospital, Zibo, China \\ Contributions: (I) Conception and design: All authors; (II) Administrative support: X Xiang, Y Xu; (III) Provision of study materials or patients: C \\ Shao; (IV) Collection and assembly of data: C Shao, Q Cheng, S Zhang; (V) Data analysis and interpretation: C Shao, Q Cheng, S Zhang; (VI) \\ Manuscript writing: All authors; (VII) Final approval of manuscript: All authors. \\ Correspondence to: Xinxin Xiang. Center of Translational Medicine, Zibo Central Hospital, Zibo 255036, China. Email: xiangxinxin1985@163.com; \\ Youqing Xu. Department of Gastroenterology, Beijing Tiantan Hospital, Capital Medical University, Beijing, China. \\ Email: youqingxutttyy@163.com.
}

\begin{abstract}
Background Non-alcoholic fatty liver disease (NAFLD) has become a major public health problem concern in recent decades. The specific mechanism of NAFLD is still not clear. Previous studies had shown the correlation between NAFLD and thyroid dysfunction. The correlation between thyroid hormones within the euthyroid range and NAFLD has not yet been clarified. This study sought to investigate the association between NAFLD and thyroid hormones in euthyroid patients.
\end{abstract}

Methods: A retrospective cross-sectional study was conducted at Beijing Tiantan Hospital from January 1, 2019, to October 1, 2021. Eighty-one NAFLD patients with normal thyroid function and 34 healthy individuals were enrolled. Participants' demographic information, biochemical parameters, and thyroid hormone levels were collected. The severity of NAFLD was assessed by abdominal computed tomography (CT). The association between NAFLD and thyroid hormones was analyzed.

Results: Patients in the NAFLD group were older and more likely to be female than those in the healthy control group $(\mathrm{P}<0.05)$. Compared to the healthy control group, the serum levels of fasting plasma glucose (FPG), alanine transaminase (ALT), plasma aspartate transaminase (AST), triglyceride, gammaglutamyl transferase $(\gamma-\mathrm{GT})$, and uric acid (UA) were higher, but the levels of high-density lipoprotein cholesterol (HDL-C), and free thyroxine $\left(\mathrm{FT}_{4}\right)$ were lower in the NAFLD group $(\mathrm{P}<0.05)$. NAFLD is more severe in females than males $(\mathrm{P}<0.05)$. ALT, AST, low-density lipoprotein cholesterol (LDL-C), $\gamma$-GT, tetraiodothyronine, and free triiodothyronine $\left(\mathrm{FT}_{3}\right)$ levels increased significantly as the severity of NAFLD increased $(\mathrm{P}<0.05)$. The results of the Spearman correlation analysis indicated that the severity of NAFLD was positively correlated with ALT ( $\mathrm{r}=0.376, \mathrm{P}=0.001)$, AST $(\mathrm{r}=0.275, \mathrm{P}=0.015)$, and LDL $(\mathrm{r}=0.313, \mathrm{P}=0.007)$. The multiple logistic regression analysis showed that age [odds ratio $(\mathrm{OR})=1.071 ; 95 \%$ confidence interval (CI): 1.010-1.136, P=0.021], ALT (OR =1.091; 95\% CI: 1.034-1.150, P=0.001), HDL-C $(\mathrm{OR}=0.085 ; 95 \% \mathrm{CI}: 0.010-0.690, \mathrm{P}=0.021)$, and $\mathrm{FT}_{4}(\mathrm{OR}=0.738 ; 95 \% \mathrm{CI}: 0.545-1.001, \mathrm{P}=0.046)$ were independently related to the risk of NAFLD in patients with normal thyroid function.

Conclusions: $\mathrm{FT}_{4}$ within the normal range was lower in the NAFLD group compared to the healthy control group. The serum level of $\mathrm{FT}_{4}$ is an independent risk factor of NAFLD in euthyroid people.

Keywords: Euthyroid; non-alcoholic fatty liver disease (NAFLD); thyroid hormones

Submitted Dec 09, 2021. Accepted for publication Feb 14, 2022.

doi: 10.21037/apm-21-3890

View this article at: https://dx.doi.org/10.21037/apm-21-3890 


\section{Introduction}

Nonalcoholic fatty liver disease (NAFLD) is a clinical and pathological syndrome caused by pathologic factors except alcohol or other known factors that injure liver (1). The main characteristic of NAFLD is fatty infiltration in $>5 \%$ of hepatocytes (2). The disease spectrum of NAFLD includes benign non-alcoholic fatty liver, and the more severe nonalcoholic steatohepatitis (NASH), which is characterized by steatosis, hepatocellular ballooning, lobular inflammation, and almost always fibrosis, and can progress to liver cirrhosis and hepatocellular carcinoma (3). With changes in lifestyle and improvements in people's living standards, NAFLD has become an important cause of chronic liver disease worldwide, and has a prevalence rate of $25-30 \%$ in the general population $(4,5)$. The economic burden of NAFLD has been projected to be approximately $>100$ billion dollars in the United States and about 35 billion dollars in Italy, Germany, the United Kingdom, and France (6). Presently, the prevalence rate of NAFLD in China is $29.2 \%$. As a result of urbanization, it is estimated that by 2030 there will be 315 million NAFLD patients in China, and China will have the highest growth in the prevalence of NAFLD globally $(7,8)$.

Thyroid hormones are important hormones in maintaining metabolic homeostasis, and regulate de novo lipogenesis, fatty acid oxidation, cholesterol metabolism, and carbohydrate metabolism, and play important roles in hepatic lipid metabolism (9). Sinha et al. reported that hypothyroidism is associated with increased serum levels of triglycerides, cholesterol, and NAFLD (10). A large and updated meta-analysis found that hypothyroidism is significantly associated with the presence and severity of NAFLD (11). Ludwig et al. confirmed the association between hypothyroidism (both subclinical and clinical) and hepatic steatosis in NAFLD (12). There have been many studies on NAFLD and thyroid hormone. But the correlation between thyroid hormones within the euthyroid range and NAFLD has not yet been clarified. At present, fewer studies have explored the association between thyroid hormones and the severity of NAFLD in euthyroid subjects. In this study, we investigated the relationship between thyroid hormones and the occurrence and severity NAFLD in euthyroid individuals to provide insights into new predictors and treatment targets for NAFLD.

We present the following article in accordance with the STROBE reporting checklist (available at https://apm. amegroups.com/article/view/10.21037/apm-21-3890/rc).

\section{Methods}

\section{Study population}

This retrospective cross-sectional study was conducted at Beijing Tiantan Hospital from January 1, 2019, to October 1, 2021. Eighty-one NAFLD patients with normal thyroid function and 34 healthy individuals were enrolled in the study. NAFLD was diagnosed according to the 2018 guidelines for the prevention and treatment of NAFLD (13).

All participants underwent abdominal computed tomography (CT). Subjects were excluded if they met any of the following exclusion criteria: (I) were aged $<18$ years; (II) engaged in significant alcohol consumption ( $>20 \mathrm{~g} /$ day for women and $>30 \mathrm{~g} /$ day for men); (III) had liver disease caused by virus, alcohol, drug, autoimmunity, and/or total parenteral nutrition; (IV) had established hypertension, coronary heart disease, diabetes, hyperthyroidism, hypothyroidism, acute and chronic renal insufficiency, a tumor, or other serious acute and chronic diseases; and/ or $(\mathrm{V})$ had been treated with anti-thyroid drugs, thyroid hormone drugs, amiodarone, and/or other drugs affecting thyroid function.

The study was conducted in accordance with the Declaration of Helsinki (as revised in 2013). The study was approved by Medical Ethics Committee of Beijing Tiantan Hospital (No. KY2016-042-01) and individual consent for this retrospective analysis was waived.

\section{Clinical and laboratory data collection}

Participants' demographic information (age and sex) was collected. Biochemical parameters, including fasting plasma glucose (FPG), alanine transaminase (ALT), plasma aspartate transaminase (AST), gamma-glutamyl transferase $(\gamma-\mathrm{GT})$, alkaline phosphatase (ALP), total bilirubin (TBIL) and indirect bilirubin (IBIL), triglyceride (TG), total cholesterol (TC), high-density lipoprotein cholesterol (HDL-C), low-density lipoprotein cholesterol (LDL-C), blood urea nitrogen (BUN), uric acid (UA) and creatinine $(\mathrm{Cr})$ were measured by a LAbOSPECT 008 automatic analyzer. Thyroid function tests, including thyroid stimulating hormone (TSH), free thyroxine $\left(\mathrm{FT}_{4}\right)$, free triiodothyronine $\left(\mathrm{FT}_{3}\right)$, triiodothyronine $\left(\mathrm{TT}_{3}\right)$ and tetraiodothyronine $\left(\mathrm{TT}_{4}\right)$ were measured by the UniCel Dxl 
800 Access automatic analyzer. All the blood samples were obtained intravenously after 8 hours of fasting.

\section{Abdominal CT evaluation}

Abdominal CT examinations were performed by Somatom Definition (Siemens Medical Solutions, Forchheim, Germany). The ratio of CT values of the liver and spleen in NAFLD is $\leq 1$. A mild degree of NAFLD is indicated by $0.7<$ liver/spleen CT value $\leq 1.0$, a moderate degree of NAFLD is indicated by $0.5<$ liver/spleen CT value $\leq 0.7$, and a severe degree of NAFLD is indicated by liver/spleen CT value $\leq 0.5$ (14).

\section{Statistical analysis}

The normally distributed continuous data are expressed as the mean \pm standard deviation (SD), and the non-normal distributed continuous variables are expressed as the median $\left(\mathrm{P}_{25}, \mathrm{P}_{75}\right)$. Comparisons of continuous data between groups were performed by the Student's $t$-test or MannWhitney $U$ test. Categorical variables were summarized in frequencies or percentages, and the $\chi^{2}$ test was used to compare groups. A Spearman rank-correlation analysis was used for the correlation analysis. Logistic regression was used to investigate the risk factors related to NAFLD. All the statistical analyses were performed using SPSS 21.0 software. A P value $<0.05$ ( 2 -tailed) was considered statistically significant.

\section{Results}

Characteristics of the study participants in the NAFLD and bealthy control groups

As Table 1 shows, patients in the NAFLD were older and more likely to be female than individuals in the healthy control group $(\mathrm{P}<0.05)$. Compared to the healthy control group, the serum levels of FPG, ALT, AST, TG, $\gamma$-GT, and UA were higher but the levels of HDL-C and $\mathrm{FT}_{4}$ were lower in the NAFLD group $(\mathrm{P}<0.05)$. There were no differences in the TC, LDL-C, Cr, TSH, $\mathrm{TT}_{3}, \mathrm{TT}_{4}$, and $\mathrm{FT}_{3}$ levels between the NAFLD and healthy control groups $(\mathrm{P}>0.05)$.

\section{Comparison of characteristics and the severity of NAFLD}

According to the abdominal CT results, NAFLD patients were divided into mild $(n=26)$, moderate $(n=24)$, and severe $(\mathrm{n}=31)$ groups. NAFLD was more severe in female patients than male patients $(\mathrm{P}<0.05)$. ALT, AST, LDL-C, $\gamma-\mathrm{GT}$, $\mathrm{TT}_{4}$, and $\mathrm{FT}_{3}$ levels increased significantly as the severity of NAFLD increased $(\mathrm{P}<0.05)$. However, no statistically significant differences in FPG, TG, TC, HDL-C, Cr, UA, $\mathrm{TSH}, \mathrm{TT}_{3}$ and $\mathrm{FT}_{4}$ levels were observed among the groups ( $\mathrm{P}>0.05$; see Table 2).

\section{Spearman correlation of the characteristics and the severity of NAFLD}

The results of the Spearman correlation analysis indicated that the severity of NAFLD was positively correlated with ALT ( $r=0.376, \mathrm{P}=0.001)$, AST $(\mathrm{r}=0.275, \mathrm{P}=0.015)$, and LDL $(\mathrm{r}=0.313, \mathrm{P}=0.007)$.

\section{Multivariate analysis}

The results of the multiple logistic regression analysis showed that age [odds ratio $(\mathrm{OR})=1.071 ; 95 \%$ confidence interval (CI): 1.010-1.136, $\mathrm{P}=0.021$ ], ALT $(\mathrm{OR}=1.091$; 95\% CI: $1.034-1.150, \mathrm{P}=0.001)$, HDL-C $(\mathrm{OR}=0.085$; 95\% CI: $0.010-0.690, \mathrm{P}=0.021)$, and $\mathrm{FT}_{4}(\mathrm{OR}=0.738 ; 95 \%$ CI: $0.545-1.001, \mathrm{P}=0.046)$ were independently related to the risk of NAFLD in participants with normal thyroid function.

\section{Discussion}

As a metabolic disease involving multiple systems, NAFLD has become a major public health concern in recent decades. With the global epidemic of obesity fueling metabolic conditions, the clinical and economic burden of NAFLD will become enormous (6). In the past 10 years, the liver transplantation rate caused by NASH has increased from $1.2 \%$ to $9.7 \%$ (15). It is estimated that NAFLD will become the leading cause of end-stage liver disease in the next decades. The specific mechanism of NAFLD is still not clear. The disease burden needs to be mitigated. As the hepatic manifestation of metabolic syndrome, NAFLD is associated with metabolic disorders, including central obesity, hyperlipidemia, diabetes, and hypertension (16).

In mammals, thyroid hormones play an important role in regulating lipid and carbohydrate metabolism, energy metabolism, and nervous system development. Thyroid hormones play an important role in maintaining liver homeostasis. Thyroid hormones have prominent effects on hepatic fatty acid and cholesterol synthesis and metabolism. 
Table 1 Characteristics of the NAFLD and healthy control groups

\begin{tabular}{|c|c|c|c|}
\hline Characteristics & NAFLD group & Healthy control group & $P$ value \\
\hline Age (years) & $44[33,54]$ & $34[27,44]$ & $<0.05$ \\
\hline Sex (male/female) & $29 / 52$ & $21 / 13$ & $<0.05$ \\
\hline FPG (mmol/L) & $5.19(4.70,6.36)$ & $5.08(4.83,5.42)$ & $<0.05$ \\
\hline AST (U/L) & $25(17.88,40.33)$ & $15.05(13.48,17.75)$ & $<0.05$ \\
\hline $\mathrm{TG}(\mathrm{mmol} / \mathrm{L})$ & $1.74(1.07,2.60)$ & $0.87(0.52,1.42)$ & $<0.05$ \\
\hline $\mathrm{TC}(\mathrm{mmol} / \mathrm{L})$ & $4.56(3.77,5.23)$ & $4.44(3.88,5.46)$ & 0.75 \\
\hline HDL-C (mmol/L) & $1.22 \pm 0.33$ & $1.59 \pm 0.32$ & $<0.01$ \\
\hline $\mathrm{Cr}(\mu \mathrm{mol} / \mathrm{L})$ & $61.63 \pm 14.46$ & $57.81 \pm 14.21$ & 0.25 \\
\hline $\mathrm{UA}(\mu \mathrm{mol} / \mathrm{L})$ & $368.83 \pm 97.64$ & $298.12 \pm 93.38$ & $<0.01$ \\
\hline $\mathrm{TSH}(\mu \mathrm{IU} / \mathrm{mL})$ & $1.77(1.17,2.34)$ & $1.82(1.16,2.68)$ & 0.99 \\
\hline $\mathrm{TT}_{3}(\mathrm{nmol} / \mathrm{L})$ & $1.52 \pm 0.27$ & $1.54 \pm 0.23$ & 0.78 \\
\hline $\mathrm{TT}_{4}(\mathrm{nmol} / \mathrm{L})$ & $105.30 \pm 16.12$ & $106.62 \pm 19.75$ & 0.06 \\
\hline $\mathrm{FT}_{3}(\mathrm{pmol} / \mathrm{L})$ & $5.13 \pm 0.67$ & $4.93 \pm 0.44$ & 0.25 \\
\hline $\mathrm{FT}_{4}(\mathrm{pmol} / \mathrm{L})$ & $11.38 \pm 1.97$ & $13.01 \pm 1.76$ & $<0.05$ \\
\hline
\end{tabular}

NAFLD, non-alcoholic fatty liver disease; FPG, fasting plasma glucose; ALT, alanine transaminase; AST, plasma aspartate transaminase; TG, triglyceride; TC, total cholesterol; HDL-C, high-density lipoprotein cholesterol; LDL-C, low-density lipoprotein cholesterol; $\gamma$-GT, gamma-glutamyl transferase; $\mathrm{Cr}$, creatinine; $\mathrm{UA}$, uric acid; $\mathrm{TSH}$, thyroid stimulating hormone; $\mathrm{FT}_{3}$, free triiodothyronine; $\mathrm{FT}_{4}$, free thyroxine; $\mathrm{TT}_{3}$, triiodothyronine; $\mathrm{TT}_{4}$, tetraiodothyronine.

It has been reported that the prevalence of NAFLD is significantly higher in subclinical hypothyroidism patients than in those with euthyroidism, and that TSH but not $\mathrm{FT}_{4}$ is independently associated with NAFLD (17). In this study, we examined the correlation between thyroid hormones and NAFLD in euthyroid individuals, and found that the NAFLD participants were older and more likely to be female than the healthy control participants. Lin et al. reported that patients with metabolic associated fatty liver disease (MAFLD) were significantly older compared to NAFLD group (18). DiStefano et al. demonstrated that postmenopausal women have a higher risk of NAFLD and higher rates of severe hepatic fibrosis (19). Recent reports have shown that the prevalence of NAFLD in women has increased over the past 10 years, and the mortality rate of women has risen sharply compared to that of men $(20,21)$. FPG, ALT, AST, TG, $\gamma$-GT, and UA serum levels were higher but HDL-C and $\mathrm{FT}_{4}$ levels were lower in the NAFLD group than the healthy control group in our study. No differences in the TC, LDL-C, Cr, TSH, TT ${ }_{3}$, $\mathrm{TT}_{4}$ and $\mathrm{FT}_{3}$ levels were observed between the NAFLD and healthy control groups. As is well known, NAFLD is associated with insulin resistance (IR) and type 2 diabetes, which could explain the high FPG level in the NAFLD group (22). Many studies have investigated whether a high UA level is independently associated with NAFLD $(23,24)$. The UA level was higher in the NAFLD participants than the healthy control participants in our study, but it was not independently related to NAFLD.

The association between thyroid hormones and the severity of NAFLD remains unclear. It is reported that altered thyroid profile was significantly associated with an increased incidence of NAFLD and low serum $\mathrm{FT}_{4}$ was the only independent predictor of assessing significant 
Table 2 Characteristics of patients with NAFLD in different severity groups

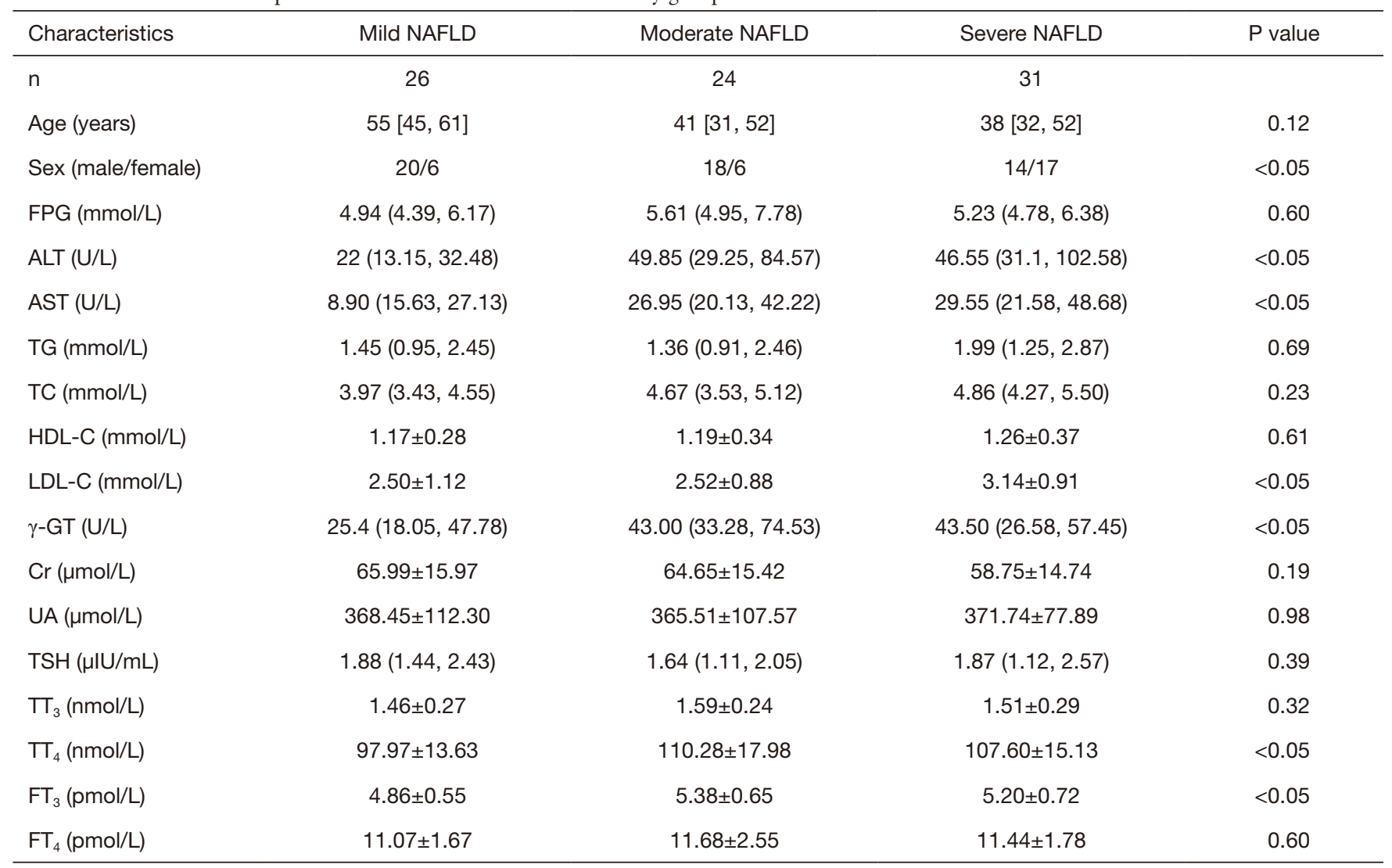

NAFLD, non-alcoholic fatty liver disease; FPG, fasting plasma glucose; ALT, alanine transaminase; AST, plasma aspartate transaminase; TG, triglyceride; TC, total cholesterol; HDL-C, high-density lipoprotein cholesterol; LDL-C, low-density lipoprotein cholesterol; $\gamma$-GT, gamma-glutamyl transferase; $\mathrm{Cr}$, creatinine; $\mathrm{UA}$, uric acid; $\mathrm{TSH}$, thyroid stimulating hormone; $\mathrm{FT}_{3}$, free triiodothyronine; $\mathrm{FT}_{4}$, free thyroxine; $\Pi_{3}$, triiodothyronine; $\mathrm{TT}_{4}$, tetraiodothyronine.

fibrosis in NAFLD participants (25). Tahara et al. found that TSH level was positively associated with the progression of liver fibrosis (17). Kim et al. reported that subclinical hypothyroidism is an independent predictor of histological NASH and advanced fibrosis (26). Manka suggested that lower $\mathrm{FT}_{3}$ and $\mathrm{FT}_{4}$ were associated with higher liver stiffness and a higher NAFLD fibrosis score, respectively (27). Guo et al. reported that $\mathrm{FT}_{3}$ level is positively correlated with NAFLD severity in euthyroid participants (28). Tao et al. showed TSH and $\mathrm{FT}_{4}$ levels were independent risk factors for NAFLD in euthyroid general subjects. However, their research did not study the association between thyroid hormones and the severity of NAFLD (29). In our study, the NAFLD patients were divided into mild, moderate and severe groups according to their abdominal CT results. NAFLD was found to be more severe in females than males, and the ALT, AST, LDL-C,
$\gamma$-GT, $\mathrm{TT}_{4}$ and $\mathrm{FT}_{3}$ levels significantly increased as the severity of NAFLD increased. However, only the ALT, AST and LDL levels were positively correlated with the severity of NAFLD according to the Spearman analysis. Our results suggest that thyroid hormones levels in the normal range were not related to the severity of NAFLD.

Jaruvongvanich et al. found no association between NAFLD and thyroid hormone (30). Another meta-analysis investigated the correlation between NAFLD and thyroid function (31). He et al. showed that individuals with subclinical and overt hypothyroidism are at higher risk for NAFLD than euthyroid subjects (32). Bano et al. observed that lower $\mathrm{FT}_{4}$ and higher TSH levels are associated with an increased risk of NAFLD (33). Tan et al. found that a high TSH level was significantly associated with NAFLD in T2DM patients with euthyroid function (34). Huang et al. reported that high levels of $\mathrm{FT}_{3}$ and TSH increased the risk 
of NAFLD in euthyroid patients with T2DM (35). The present study showed that only $\mathrm{FT}_{4}$ within the reference range was an independent risk factor of NAFLD. Xu et al. also reported that serum $\mathrm{FT}_{4}$ level was significantly associated with the risk for NAFLD in elderly Chinese euthyroid participants (36). Liu et al. investigated that high levels of $\mathrm{FT}_{3}$ were significantly associated with NAFLD among middle-aged euthyroid subjects independently of known metabolic risk factors, while only serum $\mathrm{FT}_{4}$ levels were negatively associated with NAFLD in pre-menopausal women (37). The conflicting results may be caused by many reasons. Diagnostic accuracy of ultrasonography is inferior to pathological diagnosis by liver biopsy (38). It is reported that menopausal status could change the interactions between serum $\mathrm{FT}_{4}$ level and metabolic syndrome components (39). Multi-center clinical trials are more conducive to reduce the deviation and obtain more definite results.

In conclusion, the major finding of our cross-sectional study was that $\mathrm{FT}_{4}$ within the normal range was lower in the NAFLD group than the healthy control group. The $\mathrm{TT}_{4}$ and $\mathrm{FT}_{3}$ levels increased significantly as in the severity of increased. The serum level of $\mathrm{FT}_{4}$ was an independent risk factor of NAFLD in euthyroid participants. These results could provide novel insights into the prediction and treatment of NAFLD.

\section{Acknowledgments}

Funding: This work was supported by the National Science and Technology Major Project (No. 81570536), the Natural Science Foundation of Shandong Province (No. ZR2020MC062), the Key Research and Development project of Shandong Province program (No. 2019GSF108269), and the Youth Scientific Research Foundation of Beijing Tiantan Hospital (No. 2018-YQN-14).

\section{Footnote}

Reporting Checklist: The authors have completed the STROBE reporting checklist. Available at https://apm. amegroups.com/article/view/10.21037/apm-21-3890/rc

Data Sharing Statement: Available at https://apm.amegroups. com/article/view/10.21037/apm-21-3890/dss

Conflicts of Interest: All authors have completed the ICMJE uniform disclosure form (available at https://apm. amegroups.com/article/view/10.21037/apm-21-3890/coif). The authors have no conflicts of interest to declare.

Ethical Statement: The authors are accountable for all aspects of the work, including ensuring that any questions related to the accuracy or integrity of any part of the work have been appropriately investigated and resolved. The study was conducted in accordance with the Declaration of Helsinki (as revised in 2013). The study was approved by Medical Ethics Committee of Beijing Tiantan Hospital (No. KY2016-04201 ) and individual consent for this retrospective analysis was waived.

Open Access Statement: This is an Open Access article distributed in accordance with the Creative Commons Attribution-NonCommercial-NoDerivs 4.0 International License (CC BY-NC-ND 4.0), which permits the noncommercial replication and distribution of the article with the strict proviso that no changes or edits are made and the original work is properly cited (including links to both the formal publication through the relevant DOI and the license). See: https://creativecommons.org/licenses/by-nc-nd/4.0/.

\section{References}

1. Zeng MD, Fan JG, Lu LG, et al. Guidelines for the diagnosis and treatment of nonalcoholic fatty liver diseases. J Dig Dis 2008;9:108-12.

2. Sanyal AJ, Brunt EM, Kleiner DE, et al. Endpoints and clinical trial design for nonalcoholic steatohepatitis. Hepatology 2011;54:344-53.

3. Kleiner DE, Makhlouf HR. Histology of Nonalcoholic Fatty Liver Disease and Nonalcoholic Steatohepatitis in Adults and Children. Clin Liver Dis 2016;20:293-312.

4. Bellentani S. The epidemiology of non-alcoholic fatty liver disease. Liver Int 2017;37 Suppl 1:81-4.

5. Chalasani N, Younossi Z, Lavine JE, et al. The diagnosis and management of nonalcoholic fatty liver disease: Practice guidance from the American Association for the Study of Liver Diseases. Hepatology 2018;67:328-57.

6. Younossi ZM, Koenig AB, Abdelatif D, et al. Global epidemiology of nonalcoholic fatty liver disease-Metaanalytic assessment of prevalence, incidence, and outcomes. Hepatology 2016;64:73-84.

7. Zhou F, Zhou J, Wang W, et al. Unexpected Rapid Increase in the Burden of NAFLD in China From 2008 to 2018: A Systematic Review and Meta-Analysis. Hepatology 2019;70:1119-33. 
8. Estes C, Anstee QM, Arias-Loste MT, et al. Modeling NAFLD disease burden in China, France, Germany, Italy, Japan, Spain, United Kingdom, and United States for the period 2016-2030. J Hepatol 2018;69:896-904.

9. Ritter MJ, Amano I, Hollenberg AN. Thyroid Hormone Signaling and the Liver. Hepatology 2020;72:742-52.

10. Sinha RA, Singh BK, Yen PM. Direct effects of thyroid hormones on hepatic lipid metabolism. Nat Rev Endocrinol 2018;14:259-69.

11. Mantovani A, Nascimbeni F, Lonardo A, et al. Association Between Primary Hypothyroidism and Nonalcoholic Fatty Liver Disease: A Systematic Review and Meta-Analysis. Thyroid 2018;28:1270-84.

12. Ludwig U, Holzner D, Denzer C, et al. Subclinical and clinical hypothyroidism and non-alcoholic fatty liver disease: a cross-sectional study of a random population sample aged 18 to 65 years. BMC Endocr Disord 2015;15:41.

13. National Workshop on Fatty Liver and Alcoholic Liver Disease, Chinese Society of Hepatology, Chinese Medical Association; Fatty Liver Expert Committee, Chinese Medical Doctor Association. Guidelines of prevention and treatment for nonalcoholic fatty liver disease: a 2018 update. Zhonghua Gan Zang Bing Za Zhi 2018;26:195-203.

14. Ataseven H, Yildirim MH, Yalniz M, et al. The value of ultrasonography and computerized tomography in estimating the histopathological severity of nonalcoholic steatohepatitis. Acta Gastroenterol Belg 2005;68:221-5.

15. Marcuccilli M, Chonchol M. NAFLD and Chronic Kidney Disease. Int J Mol Sci 2016;17:562.

16. Roeb E, Geier A. Nonalcoholic steatohepatitis (NASH) - current treatment recommendations and future developments. Z Gastroenterol 2019;57:508-17.

17. Tahara K, Akahane T, Namisaki T, et al. Thyroidstimulating hormone is an independent risk factor of nonalcoholic fatty liver disease. JGH Open 2019;4:400-4.

18. Lin S, Huang J, Wang M, et al. Comparison of MAFLD and NAFLD diagnostic criteria in real world. Liver Int 2020;40:2082-9.

19. DiStefano JK. NAFLD and NASH in Postmenopausal Women: Implications for Diagnosis and Treatment. Endocrinology 2020;161:bqaa134.

20. Paik JM, Henry L, De Avila L, et al. Mortality Related to Nonalcoholic Fatty Liver Disease Is Increasing in the United States. Hepatol Commun 2019;3:1459-71.

21. Arshad T, Golabi P, Paik J, et al. Prevalence of Nonalcoholic Fatty Liver Disease in the Female Population. Hepatol Commun 2018;3:74-83.
22. Watt MJ, Miotto PM, De Nardo W, et al. The Liver as an Endocrine Organ-Linking NAFLD and Insulin Resistance. Endocr Rev 2019;40:1367-93.

23. Di Bonito P, Valerio G, Licenziati MR, et al. High uric acid, reduced glomerular filtration rate and non-alcoholic fatty liver in young people with obesity. J Endocrinol Invest 2020;43:461-8.

24. Oral A, Sahin T, Turker F, et al. Relationship Between Serum Uric Acid Levels and Nonalcoholic Fatty Liver Disease in Non-Obese Patients. Medicina (Kaunas) 2019;55:600.

25. Zhang X, Zhang J, Dai Y, Qin J. Serum Thyroid Hormones Levels are Significantly Associated with Nonalcoholic Fatty Liver Disease in Euthyroid Chinese Population. Clin Lab. 2020. doi:10.7754/Clin. Lab.2020.200219.

26. Kim D, Kim W, Joo SK, et al. Subclinical Hypothyroidism and Low-Normal Thyroid Function Are Associated With Nonalcoholic Steatohepatitis and Fibrosis. Clin Gastroenterol Hepatol 2018;16:123-131.e1.

27. Manka P, Bechmann L, Best J, et al. Low Free Triiodothyronine Is Associated with Advanced Fibrosis in Patients at High Risk for Nonalcoholic Steatohepatitis. Dig Dis Sci 2019;64:2351-8.

28. Guo W, Qin P, Li XN, et al. Free Triiodothyronine Is Associated With Hepatic Steatosis and Liver Stiffness in Euthyroid Chinese Adults With Non-Alcoholic Fatty Liver Disease. Front Endocrinol (Lausanne) 2021;12:711956.

29. Tao $\mathrm{Y}, \mathrm{Gu} \mathrm{H}, \mathrm{Wu}$ J, et al. Thyroid function is associated with non-alcoholic fatty liver disease in euthyroid subjects. Endocr Res 2015;40:74-8.

30. Jaruvongvanich V, Sanguankeo A, Upala S. Nonalcoholic Fatty Liver Disease Is Not Associated with Thyroid Hormone Levels and Hypothyroidism: A Systematic Review and Meta-Analysis. Eur Thyroid J 2017;6:208-15.

31. Guo Z, Li M, Han B, et al. Association of non-alcoholic fatty liver disease with thyroid function: A systematic review and meta-analysis. Dig Liver Dis 2018;50:1153-62.

32. He W, An X, Li L, et al. Relationship between Hypothyroidism and Non-Alcoholic Fatty Liver Disease: A Systematic Review and Meta-analysis. Front Endocrinol (Lausanne) 2017;8:335.

33. Bano A, Chaker L, Plompen EP, et al. Thyroid Function and the Risk of Nonalcoholic Fatty Liver Disease: The Rotterdam Study. J Clin Endocrinol Metab 2016;101:3204-11.

34. Tan Y, Tang X, Mu P, et al. High-Normal Serum Thyrotropin Levels Increased the Risk of Non-Alcoholic 
Fatty Liver Disease in Euthyroid Subjects with Type 2 Diabetes. Diabetes Metab Syndr Obes 2021;14:2841-9.

35. Huang B, Yang S, Ye S. Association between Thyroid Function and Nonalcoholic Fatty Liver Disease in Euthyroid Type 2 Diabetes Patients. J Diabetes Res 2020;2020:6538208.

36. Xu C, Xu L, Yu C, et al. Association between thyroid function and nonalcoholic fatty liver disease in euthyroid elderly Chinese. Clin Endocrinol (Oxf) 2011;75:240-6.

37. Liu G, Zheng X, Guan L, et al. Free triiodothyronine levels are positively associated with non-alcoholic fatty

Cite this article as: Shao C, Cheng Q, Zhang S, Xiang X, Xu Y. Serum level of free thyroxine is an independent risk factor for non-alcoholic fatty liver disease in euthyroid people. Ann Palliat Med 2022;11(2):655-662. doi: 10.21037/apm-21-3890 liver disease in euthyroid middle-aged subjects. Endocr Res 2015;40:188-93.

38. Sanyal AJ; American Gastroenterological Association. AGA technical review on nonalcoholic fatty liver disease. Gastroenterology 2002;123:1705-25.

39. Kim BJ, Kim TY, Koh JM, et al. Relationship between serum free T4 (FT4) levels and metabolic syndrome (MS) and its components in healthy euthyroid subjects. Clin Endocrinol (Oxf) 2009; 70:152-60.

(English Language Editor: L. Huleatt) 